\title{
OPERACIÓN EFICIENTE DEL MOTOR ELÉCTRICO ASÍNCRONO DE JAULA COMO GENERADOR
}

\section{EFFICIENT OPERATION OF THE CAGE ASYNCHRONOUS MOTOR GENERATOR}

\author{
Augusto Baldeón De la Rosa ${ }^{19}$, Jorge Cairo Hurtado, Pedro Torres Mayta ${ }^{20}$, Gianfranco Medina \\ Sánchez y Yonathan Vilcahuamán Rodríguez \\ Facultad de Ingeniería Eléctrica y Electrónica
}

\section{RESUMEN}

El principal objetivo de este trabajo fue modelar la operación del motor de inducción como generador, empleando recursos de máquinas disponibles en potencias máximas menores de $180 \mathrm{~W}$. El proyecto se llevó a cabo mediante la implementación de prácticas de laboratorio, en las que se realizaron diversas pruebas, para medir parámetros como corriente, potencia, tensión y otros, para luego analizar los resultados. La primera parte consistió en modelar la máquina de inducción para distintas condiciones de operación. Luego se realizó una comparación en términos de eficiencia entre el modo motor y el generador, para determinar las ventajas y desventajas de ambos. Finalmente se analizó el motor de inducción como generador a distintas velocidades y se obtuvo la velocidad y la potencia de salida en la que se tuvo la mayor eficiencia. Entre las principales conclusiones estuvieron: determinar que el modo motor es el más eficiente en la máquina de inducción, se encontró que para velocidades de generación mayores 0 iguales $1950 \mathrm{rev} / \mathrm{min}$ el generador tiende a mantener la eficiencia en un $70 \%$. Además, se halló una diferencia entre el modelo del circuito equivalente y el real de $20 \%$ en términos de eficiencia.

Palabras clave: eficiencia, generadores de inducción conectados a la red, las máquinas de inducción.

\section{ABSTRACT}

The main objective of this study was to model the operation of the induction motor as a generator. Using resources available in machines under maximum power of 180 W., The project was carried out through laboratory practices, which conducted several tests to measure parameters such as current, power, voltage and others, and then results were analyzed. The first part consisted on modeling the induction machine different operating conditions. Then a comparison in terms of efficiency between the motor and generator mode was assessed to determine the advantages and disadvantages of both. Finally, the induction motor as a generator at different speeds was examined and the speed and power output were obtained; this last one had the highest efficiency. The main findings were: to determine which mode is the most efficient engine in the induction machine, and it was found that the one for speeds greater than or equal in generating $1950 \mathrm{rpm}$ tends to maintain efficiency by $70 \%$. A difference between the equivalent circuit model and the real one at $20 \%$ in terms of efficiency was found.

Key words: efficiency, grid-connected, induction generators, induction machines.

\section{INTRODUCCIÓN}

El proyecto consiste en el estudio de la máquina de inducción, específicamente en la aplicación del motor de inducción como generador, así

\footnotetext{
19 raul_baldeon1@yahoo.es

${ }^{20}$ petorremn@hotmail.com
} 
como el diseño de modificaciones de los parámetros del mismo para la optimización de su eficiencia. Se realiza una comparación experimental entre el motor y el generador, para comprender la implicancia de usar un motor como generador. La creciente demanda energética en la actualidad, de las pocas fuentes de energía, han creado la necesidad de innovar nuevas tecnologías de producción así como el crecimiento de las microgeneradoras, de estas tendencias el uso del motor de inducción como generador es una opción fiable, debido a su poca complejidad, tamaño y bajo precio. El diseño de las modificaciones a los parámetros de la máquina, para optimizar la eficiencia, es una idea novedosa que trata de mejorar la microgeneración. Para la realización del proyecto se plantearon objetivos como: modelar y probar un motor de inducción como generador para optimizar su operación a determinada velocidad y potencia de salida, para la aplicación en sistemas de microgeneración, desagregándose en las siguientes acciones: Modelar la operación del motor de inducción como generador, en un intervalo de potencias cercanas a $180 \mathrm{~W}$. Diseñar las modificaciones necesarias para el motor de inducción para optimizar su eficiencia en función generadora. Determinar experimentalmente la potencia y la velocidad de salida de un motor de inducción usado como generador para su máxima eficiencia. Realizar un análisis comparativo de la eficiencia de la máquina de inducción entre el modo motor y el modo generador

\section{MATERIAL Y MÉTODOS}

\section{Material}

Los materiales que se vienen empleando son los sistemas de información bibliográfica, ventanas de Internet, artículos científicos, normalizaciones, textos, software, equipos de cómputo, módulos de máquinas eléctricas en corriente continuaalterna, instrumentos de medida, y materiales de escritorio.

\section{Metodología}

El desarrollo del proyecto se rige en la siguiente metodología, se realiza una búsqueda exhaustiva de información en recursos informativos como internet y libros, relacionada con la máquina de inducción y su aplicación en la generación eléctrica, eólica 0 hidráulica. Se elabora un marco teórico, para definir los conceptos relacionados con el tema del proyecto, así como para delimitarlo.

Con la aplicación de un laboratorio se analiza la eficiencia del motor y del generador, se realizan las respectivas gráficas y se compara el comportamiento entre ellos. Estudiando los conceptos teóricos combinado con pruebas experimentales se diseñan las modificaciones de los parámetros del motor que conllevan a una mejora en su eficiencia. Con los resultados básicos se procede a realizar las conclusiones y las recomendaciones, finalizando así el trabajo. Para conseguir esto se realizó los métodos descriptivo, comparativo (análisis y síntesis) y experimental teniéndose en cuenta al diseño metodológico empleado lo siguiente: población y muestra; se cubrió el entorno local, regional o nacional, como muestra seleccionada. Técnicas y procedimientos de recolección de datos: la recopilación de la información faltante a la bibliografía común se tomó en cuenta la experiencia de los autores en los conocimientos de diferentes centros de generación hidráulica de energía eléctrica alrededor de la muestra. Técnicas de procesamiento y análisis de datos: se efectuó un procesamiento de análisis cualitativo a los datos medidos y técnicamente cuantificables.

\section{RESULTADOS Y DISCUSIÓN}

Circuito equivalente de la máquina. 
Para poder realizar el análisis, es necesario contar con el circuito equivalente de la máquina en estudio, para ello se realizaron las pruebas de rotor bloqueado, prueba de vacío y de corriente continua.

\section{Prueba de vacío.}

Se conectó la máquina de inducción como motor a la red, y se mantuvo sin carga para obtener la condición de vacío. Se aplicó la tensión nominal en terminales y se hicieron las lecturas de corriente de línea y potencia activa consumida.

\section{Prueba de corriente continua.}

Se aplicó una tensión en corriente continua en los devanados de la máquina, y se ajustó de manera que circulara la corriente nominal en ellos, esto para calentar los devanados lo más parecido posible a la operación normal.

\section{Prueba de rotor bloqueado.}

Con la ayuda de un freno magnético, se bloquea el rotor del motor, y se aplica un voltaje reducido, para obtener una corriente aproximada a la nominal.

Ya calculado los parámetros, nada más queda mostrar el circuito equivalente de la máquina el cual nos ayudará a analizar mejor el desempeño de esta.

Figura 3.1 Circuito equivalente de la máquina en estudio.

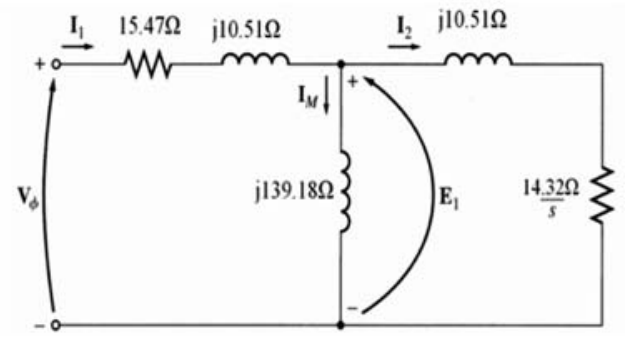

\section{Motor de corriente continua.}

Como primotor utilizado en este proyecto se equipó de una máquina de corriente continua, ya que esta permite tener varias velocidades de salida para un mismo par. Para analizar la eficiencia del generador de inducción es necesario separar las pérdidas del primotor, como no es posible acoplar el electrodinamómetro y el generador al mismo tiempo al motor, para calcular la potencia mecánica entregada al generador, se requirió de pruebas al motor a diferentes pares, y con esto tener su comportamiento a una determinada velocidad y potencia de entrada.

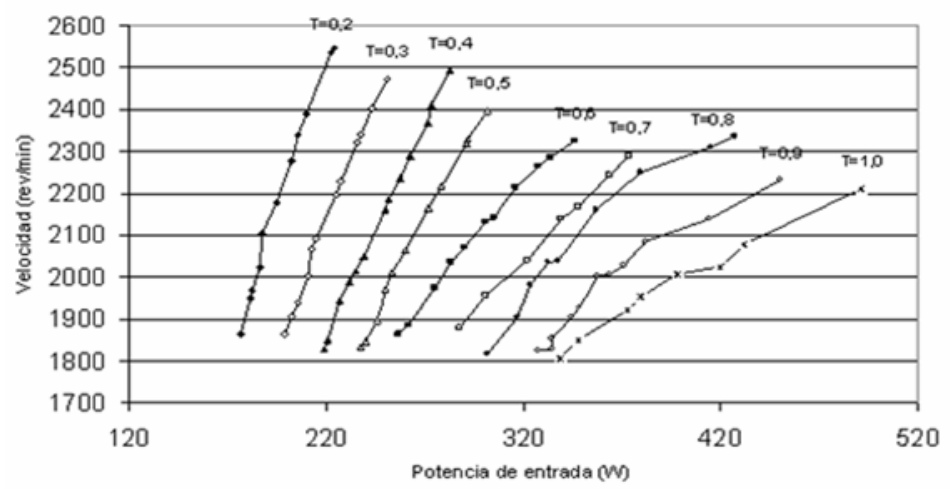

Figura 3.2 Velocidad en función de la potencia de entrada para distintos pares de carga en el motor CC. 
Figura 3.2. Es de gran ayuda, ya que con ella se podrá obtener el par de salida del motor de corriente continua, y con ello la potencia mecánica entregada por éste. Todo esto, conociendo la potencia eléctrica consumida por el motor, la velocidad, y realizando una extrapolación en la figura. Ahora, se conoce la potencia de entrada del generador y el cálculo de eficiencia del generador se puede obtener fácilmente. Como se puede entender, conforme se aumenta el par, la eficiencia aumenta, esto es debido a que se incrementa la potencia mecánica, lo que causa un incremento en potencia activa de la máquina, y una reducción en las pérdidas reactivas del campo magnético, lo que causa un mejor factor de potencia y por ende una mejora en la eficiencia. Además, se puede ver que conforme se incrementa el par las líneas tienen un rango de potencias de entrada mayor, esto se debe a que el rango de velocidades para cada par es el mismo [1800-2000rev/min] aproximadamente, pero el par es distinto, lo que causa que el intervalo de potencias sea mayor para un caso que para otro por la relación: $P \square \square \square \square$, para $0.1 \mathrm{~N}^{*} \mathrm{~m}$ la potencia de salida estará en un rango de [18.85-20.94W], y en cambio para un par de $0.3 \mathrm{~N}^{*} \mathrm{~m}$ el rango sería de [56.54-62.83W], es por eso que hay un mayor rango de potencias de entrada conforme se incrementa el par. Se puede ver también, que la pendiente de las líneas son positivas antes del par de $0.6 \mathrm{~N}^{*} \mathrm{~m}$, y a partir de ahí la pendiente de las curvas es negativa, esto es causa de la saturación de la máquina, ya que aunque se aumente la potencia eléctrica de la máquina, la potencia mecánica ya no aumentará, causando que la eficiencia disminuya en lugar de aumentar. Es importante que la eficiencia de este motor no es muy buena, ya que en el mejor de los casos superó levemente apenas el $50 \%$, lo cual indica que la mitad de la energía se consume en pérdidas, en una micro-generadora, se deben buscar turbinas que presenten una alta eficiencia, para maximizar el aprovechamiento de los recursos y hacer lo más rentable posible la planta.

\subsection{Generador de inducción}

En la figura 3.3 se observa la configuración utilizada para realizar las pruebas, se observa el módulo de adquisición de datos, el cual permitió obtener digitalmente las variables. Es importante resaltar, que la generación fue realizada conectando el generador a la red infinita y no de forma aislada.

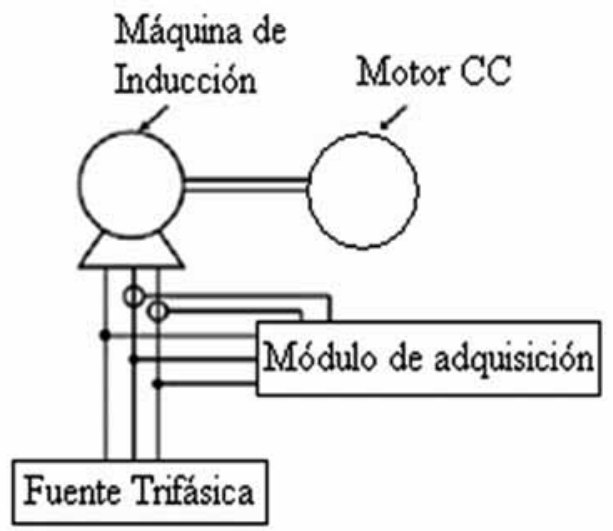

Figura 3.3 Acople de las máquinas en generación.

Se procedió a obtener las medidas como corriente y tensión para varias velocidades en el motor de corriente continua, esto con la variación de la resistencia de reóstato, y además, para distintas alimentaciones en el motor CC: reducido en un $10 \%$, reducido en un $2 \%$ y a tensión plena $(139 \mathrm{~V})$, las alimentaciones anteriores se eligieron para tener varios comportamientos, no se usó ningún criterio en especial en la elección de estas tensiones.

En esta parte, se estudiarán las ventajas y desventajas de utilizar un motor como generador, y se determinará que tan eficiente es al operar de 


\section{Prospectiva Universitaria}

esta forma. Para comenzar el análisis y discusión se hizo la prueba de vacío en la máquina, para lo cual se varío la tensión de alimentación de la máquina desde 0.1 p.u hasta 1 p.u, y se tomaron las lecturas de la corriente de armadura para cada valor de tensión.

De la curva característica sin carga, de la máquina probada. Se puede apreciar que el nivel de saturación aumenta a tensiones por encima de la tensión nominal, el nivel de saturación de la máquina afecta directamente la eficiencia, en máquinas con alta eficiencia la saturación magnética es muy pequeña, si la saturación es muy alta, una pequeña variación en la tensión provocaría un gran aumento en la corriente de magnetización, aumentando así las pérdidas. Para disminuir el nivel de saturación en la máquina, se varía el diseño de la máquina y se mejoran los materiales utilizados. La figura 4.1 compara el factor de potencia del modo motor y el modo generador para la misma corriente de armadura.

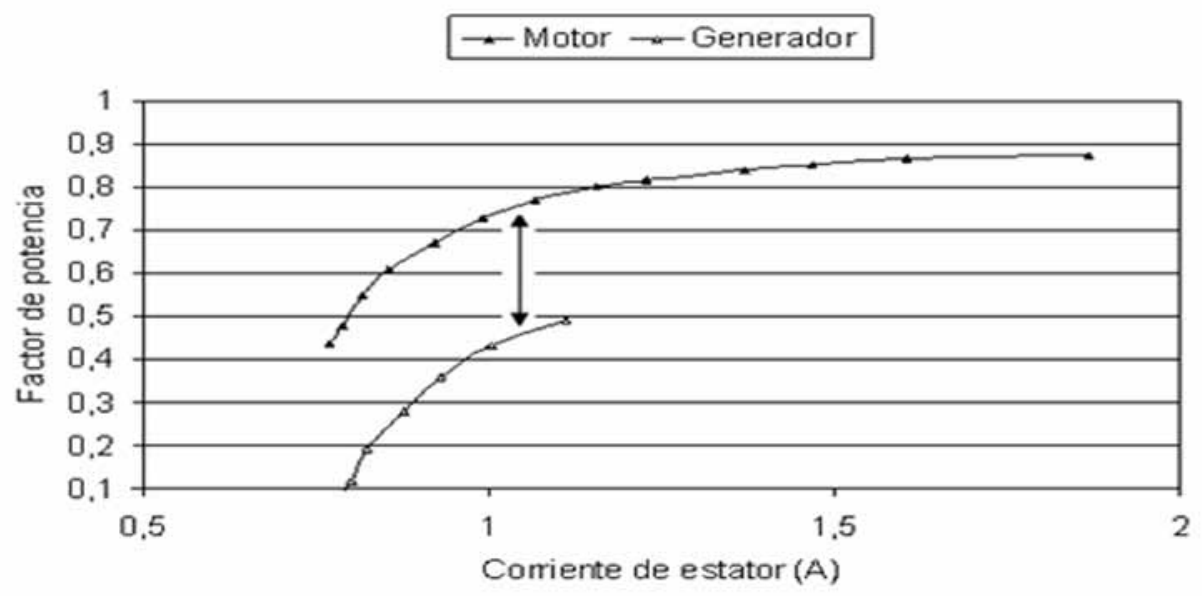

Figura 4.1 Característica del factor de potencia para ambos modos.

Como lo indica la flecha, la saturación causa que la corriente del estator en modo generador aumente debido a que la reactancia de magnetización aumente en comparación al modo motor, esto provoca que la corriente siempre sea mayor en el generador, y de naturaleza reactiva, por consiguiente, las pérdidas en el núcleo son superiores a las obtenidas en el motor. Debido a que la eficiencia es determinada a partir de la potencia activa, las pérdidas en el estator y el rotor para el modo generador y el modo motor, se comparan bajo un mismo parámetro el cual es la potencia eléctrica, que en el caso del motor es la potencia de entrada y en el caso del generador corresponde a la potencia de salida. La figura 4.2 muestra las pérdidas en el estator para la misma potencia eléctrica, al igual que las pérdidas en el núcleo. Se observa que el generador sigue teniendo mayores pérdidas; esto se puede mejorar usando materiales magnéticos de mayor calidad y probablemente cambiando el diseño del núcleo de la máquina. 


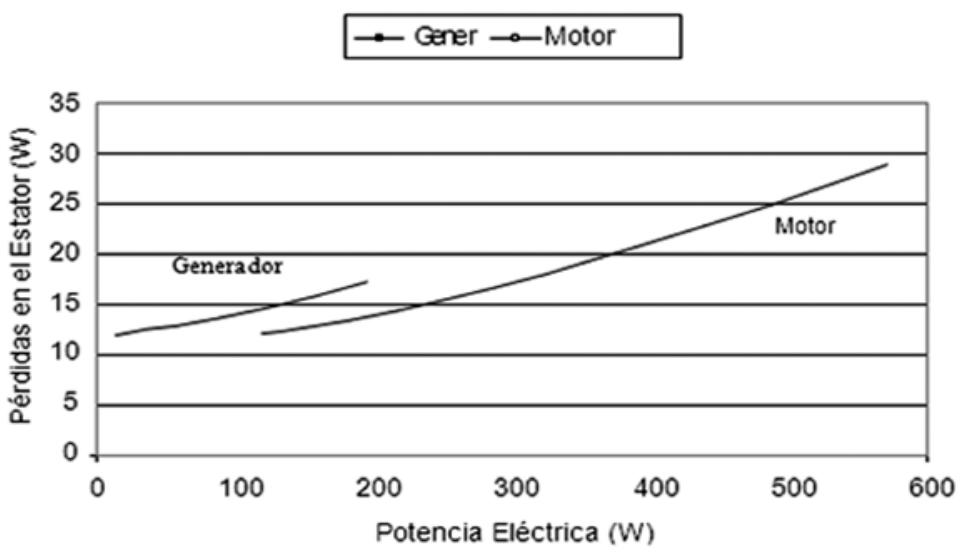

Figura 4.2 Perdidas en el estator para ambos modos en función de la potencia eléctrica.

Al igual que en las gráficas anteriores, las pérdidas en el rotor del generador superan en gran parte a las del motor, todo esto hace constatar que el modo más eficiente de operación es el motor. La diferencia en las pérdidas del estator y el rotor para el generador comparada con el motor se explican con la figura 4.2 y se asume que la potencia activa es la misma para el modo generador como para el modo como motor, en el modo motor las pérdidas activas son suplidas por la red y en el generador por la fuente mecánica. Sin embargo, la potencia reactiva para magnetizar la máquina siempre proviene de la red. Se hace uso del circuito equivalente de la máquina, donde se ubicarán los flujos de potencias y las direcciones de la corriente.

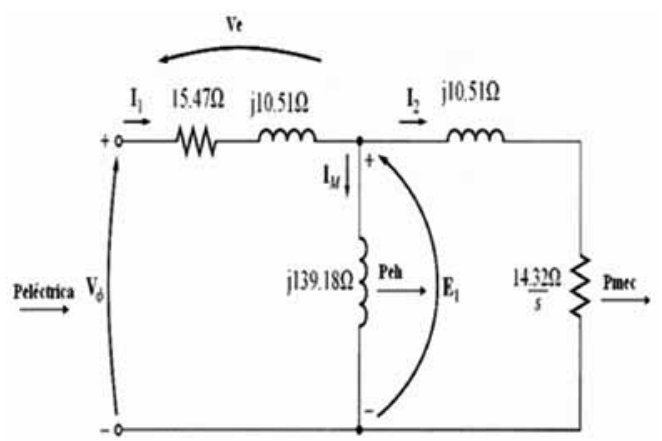

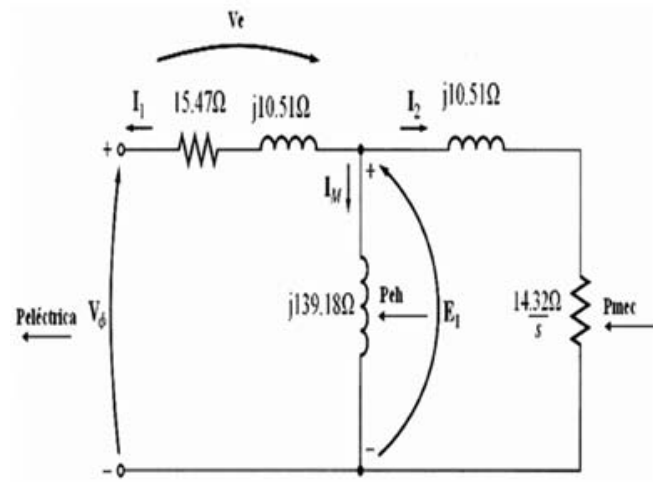

Figura 4.3 Pérdidas en el motor y generador según los flujos de potencia.

La corriente del estator y la caída de tensión en el estator incrementan con la carga, debido a que se tiene que mantener la tensión en la red la tensión en el entrehierro decrece un poco, lo que provoca que la corriente de magnetización también disminuya, y así las pérdidas en el núcleo.

En el generador, la tensión en el entrehierro se incrementa cuando se aumenta la carga, como la tensión de la red se debe mantener, cuando aumenta la tensión en el estator, la tensión en 
la rama de magnetización debe aumentar. Esto requiere una mayor corriente de magnetización y en consecuencia las pérdidas en el núcleo se incrementa. Se analizarán las pérdidas y la eficiencia en generación de la máquina para ello se tendrán dos enfoques uno desde el punto de vista del circuito equivalente y otro basado en los datos obtenidos experimentalmente. Como se puede observar las dos curvas presentan una gran similitud, lo que indica que los resultados experimentales estuvieron acertados. La diferencia entre los datos con el circuito equivalente y los experimentales, se debe a que en el circuito equivalente no se toman en cuenta diversas pérdidas como las misceláneas y las de ventilación.

El modelo del circuito equivalente se puede mejorar introduciendo una función que describa las pérdidas por ventilación y fricción que existan dependiendo de la velocidad, pues estás pérdidas son directamente proporcionales a la velocidad. Además en la práctica el calentamiento real de la máquina influye. Es importante recalcar además, que la máxima potencia obtenida fue de $194 \mathrm{~W}$, es un buen valor tomando en cuenta que la potencia nominal de la máquina es de 175W. La eficiencia es mucho mayor en el análisis con el circuito equivalente. Sin embargo la eficiencia conforme se aumenta la velocidad, deja un comportamiento aproximado lineal $y$ tiende a mantenerse constante en un valor, por ejemplo para los datos experimentales empieza a mantenerse en $70 \%$. A velocidades mayores a $1963 \mathrm{rev} / \mathrm{min}$ la eficiencia no va a aumentar, lo que lleva a la conclusión de que a partir de $1963 \mathrm{rev} / \mathrm{min}$ se obtiene la eficiencia máxima de la máquina. Con velocidades mayores a esta la potencia de salida será mayor, pero se mantendrá la eficiencia.

La principal ventaja del generador de inducción con respecto al generador sincrónico es para una misma potencia el generador de inducción posee una mayor simplicidad y un menor tamaño, sin embargo, posee una gran desventaja de que sólo puede generar potencia activa y siempre consume potencia reactiva, provocando que en su operación siempre mantenga un bajo factor de potencia, pero fácil de corregir.

Se determinó que el modo más eficiente en la máquina de inducción entre el generador y el motor, es el motor. Esto debido a que en el motor conforme aumenta la carga disminuye la corriente de magnetización, en cambio en el generador entre más carga más alta es la corriente de magnetización.

Para el generador estudiado la máxima eficiencia se encontró cuando la velocidad fue de $1963 \mathrm{rev} /$ min, teniendo una potencia de salida de $193.8 \mathrm{~W}$. Es importante señalar que la máxima eficiencia fue de $70 \%$, una eficiencia un poco baja, debido a la gran cantidad de pérdidas que se encuentran en este tipo de máquina y en la conexión del experimento.

Una ventaja del generador de inducción para su aplicación en mini hidroeléctricas o eólicas es que esta máquina puede operar dentro de un rango de deslizamiento, que para este caso fue de 0.022 a 0.091 , por lo cual el control de la velocidad es mucho más simple y económico que el control dispuesto para una máquina sincrónica.

\section{REFERENCIAS BIBLIOGRÁFICAS}

Fitzgrald, A. Máquinas eléctricas, Sexta Edición, MacGraw-Hill, México, 2004.

Chapman, J. Máquinas eléctricas. Cuarta Edición, MacGraw-Hill, México, 2005.

Wildi, T. Electrical machines, drives, and power systems, Sexta edición, Pearson Prentice Hall, EUA, 2006.

Ackermann, T. Wind Power in power systems, Primera edición, Wiley, EUA, 2005.

Irvin Kosow "Maquinas eléctricas" Reverte S.A., 
Operación eficiente del motor eléctrico asíncrono de jaula como generador
Augusto Baldeón De la Rosa, Jorge Cairo Hurtado, Pedro Torres Mayta, Gianfranco Medina Sánchez y Yonathan Vilcahuamán Rodríguez
Madrid 2005

6. A.V. Ivanov Smolenski "Maquinas Eléctricas" MIR, Mosc 1986

G. Kusic. Computer-aided power systems analysis. Prentice Hall, 1986 ISBN 0-13-164526-9 025

Antonio Gómez Expósitor, José Luis Martínez
Ramos, José Antonio Rosendo Macías, Esther Romero Ramos, Jesús Riquelme Santos, Sistemas eléctricos de potencia: problemas y ejercicios resueltos, ISBN:84-205-3558-3

Anderson P., Fouad A., Power system control and stabilility, IEEE Press, New York 1993 